\title{
Highlights
}

\section{Inclusivity enhances robustness and efficiency of social networks}

Jack Murdoch Moore, Michael Small, Gang Yan

- Network growth and rewiring models capture social inclusivity

- Inclusivity is defined using graph distance rather than labels

- Inclusivity is shown to enhance robustness and efficiency of growing or restructured networks

- The proposed growth model preserves mean degree and limiting degree distribution 


\title{
Inclusivity enhances robustness and efficiency of social networks
}

\author{
Jack Murdoch Moore ${ }^{\mathrm{a}}$, Michael Small ${ }^{\mathrm{b}, \mathrm{c}}$, Gang Yan ${ }^{\mathrm{a}, \mathrm{d}, \mathrm{e}}$ \\ ${ }^{a}$ School of Physical Science and Engineering, Tongji University, Shanghai, 200092, P. R. \\ China \\ ${ }^{b}$ Complex Systems Group, Department of Mathematics and Statistics, University of \\ Western Australia, Crawley, Western Australia 6009, Australia \\ ${ }^{c}$ Mineral Resources, CSIRO, Kensington, Western Australia 6151, Australia \\ ${ }^{d}$ Shanghai Institute of Intelligent Science and Technology, Tongji University, Shanghai, \\ 200092, P. R. China \\ ${ }^{e}$ Center for Excellence in Brain Science and Intelligence Technology, Chinese Academy \\ of Sciences, Shanghai, 200031, P. R. China
}

\begin{abstract}
Many modern institutions seek to be inclusive, but the quantitative benefits of this goal are not always communicated effectively to stakeholders. To facilitate this important dialogue, we propose a simple model with which to grow, in the presence of a given level of inclusivity, networks which represent the structure of organisations. The model proceeds via an unweighted random walk in which inclusivity $r$ is the maximum allowable separation between a new contact and the set of established contacts, which thus represents the maximum tolerable amount of novelty or "otherness". The model can capture realistic small world and scale-free properties. In addition, the model fixes the limiting degree distribution, and particular parameter choices and initial conditions also fix clustering coefficient, and so allows the role of inclusivity to be isolated from these confounding factors. By considering this model, and also by randomly rewiring real networks in either an inclusive or an exclusive way, we show that, in comparison to exclusivity, inclusivity promotes unity (by decreasing modularity), efficiency and robustness. Increasing the ratio of the number of links to the number of nodes also enhances these qualities, as well as reducing their dependence upon inclusivity.
\end{abstract}

Email address: jackmoore@tongji.edu.cn (Jack Murdoch Moore) 
Keywords: Network models, Scale-free networks, Small world networks, Inclusivity, Efficiency, Robustness

\section{Introduction}

Modern communities and organisations expend effort and resources in the pursuit of inclusivity [1, 2, 3]. Some stakeholders see a moral imperactive for inclusivity [4, 5], while others appreciate indirect benefits, such as increased productivity, creativity, growth opportunities or access to talent [6, 7, 3, 8]. However, still others see this pursuit as wasteful, unfair or even harmful [4, 2, 9, 5] and so policies designed to promote harmony can instead lead to discontent and division (For brief, journalistic discussions on particular issues pertaining to inclusivity in business see Ref. [10, 11, 12]). If society and organisations continue to seek inclusivity then, to avoid unnecessary discord, effort should be made to communicate clearly and convincingly its quantitative benefits.

In order to promote a better understanding of the role of inclusivity in organisations and society, we propose a simple graph model which grows networks using a notion of inclusivity which depends only on network structure rather than postulates of non-topological node properties. This not only leads to networks with believable properties, but also allows the role of inclusivity to be isolated and assessed. Our model grows societies represented by realistic small world and scale-free networks in the presence of a given level of inclusivity. We proceed via an unweighted random walk in which inclusivity $r$ is the maximum allowable separation between a new contact and the set of established contacts, which thus represents the maximum tolerable amount of novelty or "otherness". The model fixes the limiting degree distribution, and particular parameter choices and initial conditions also fix clustering coefficient, and so allows the role of inclusivity to be isolated from these confounding factors. We find that, for odd values of the inclusivity $r$ and for a large range of reasonable values of the number $m$ of links per incoming node, inclusivity enhances unity (by decreasing modularity), network efficiency and network robustness.

We should point out that this model is geared towards investigating inclusivity rather than the related issue of diversity. A diverse structure is one in which a wide range of groups are represented, but such a structure is only inclusive when differences do not impede connection and engagement [11, 12]. 
We assume that structures are diverse and/or grow in a way which provides diversity, and consider the extent to which individuals are willing to engage with others from whom they are different. Rather than assigning node labels whereby to assess differences between nodes, we assume that social distance provides a measure of "otherness". This choice is partly motivated by a suspicion that, although religious, biological, cultural and economic differences are inevitable, it does not seem inevitable that such differences should impede connection, and much of the perceived significance of these differences could ultimately have arisen from social distance.

The minimally inclusive form of the proposed model limits to two the distance spanned by new edges, and so leads to a triadic closure or transitivity [13] mechanism. Triadic closure can be employed in either static network models, in which the set of nodes remains constant as the model evolves, or in network growth models. Our investigation of the development of organisations and societies motivates a network growth model. In particular, for simplicity and to align with influential established network growth models, we propose one in which: (1) in each step the set of nodes is augmented by precisely one node, (2) any new edge(s) added in a time step involve the most recently added node, and (3) the set of edges does not decrease in any time step.

We have not encountered the inclusivity model in the literature, but have noticed related network growth models. Under the link selection [14, 15] model, each incoming node connects to each node involved in a randomly chosen link. The link selection model always generates networks with two links per node in the large network limit, but the Holme-Kim [16] generalises the link selection model. In the most relevant case of the Holme-Kim model, each neighbour after the first is chosen by following an edge attached to the most recently chosen neighbour, unless this would lead to a duplicate link. This restriction to neighbours of the most recently made contact distinguishes the Holme-Kim model from the minimally inclusive version of the proposed model. Random walker models [17, 18, 19, 20] involve adding links to the terminal points of random walks the initial points of which can begin either at the most recently added neighbour or a randomly chosen node. The inclusivity model differs from these random walker models because, under the inclusivity model, simultaneously: (1) new neighbours can be made via any established neighbour, not just that most recently added, and (2) when at least one neighbour has been established, connections can only be made through established neighbours. Random walker models involving fi- 
nite random walks the starting point of which is chosen uniformly at random (or according to any probability distribution other than one proportional to node degree) also differ from the proposed model because they do not guarantee linear preferential attachment and so do not condition on the limiting degree distribution, which can vary with the length of the random walk [18, 19].

In Section 2 we define some important network measures and properties. We use Section 3 to propose a network model and rewiring algorithm. In Section 4 we specify parameters and identify the real-world networks we will analyse. Numerical results are presented and discussed in Section 5, and in Section 6 we conclude.

\section{Network properties}

In this section we introduce salient network measures and properties. We consider an unweighted, undirected network of $N$ nodes, labelled $\{1,2, \ldots, N\}$, without duplicate edges or self-loops, and with adjacency matrix $\left(a_{i j}\right)$.

The mean internode distance $\langle\rho\rangle$ between distinct nodes can be written

$$
\langle\rho\rangle=\frac{1}{N(N-1)} \sum_{i=1}^{N} \sum_{\substack{j=1 \\ j \neq i}}^{N} \rho_{i, j},
$$

where $\rho_{i, j}$ is the number of edges in the shortest path between nodes $i$ and $j$. The (global) clustering coefficient [21] $C$ is the fraction of connected ordered triples of distinct nodes which induce a triangle:

$$
C=\frac{\sum_{i=1}^{N} \sum_{j=i+1}^{N} \sum_{k=j+1}^{N} a_{i j} a_{j k} a_{k i}}{\sum_{i=1}^{N} \sum_{j=i+1}^{N} \sum_{k=j+1}^{N} a_{i j} a_{j k}} .
$$

The small world property [22, 23] is defined in terms of the clustering coefficient $C$ and the mean internode distance $\langle\rho\rangle$. Specifically, a network is called small world when: (1) the scaling with size $N$ of mean internode distance $\langle\rho\rangle$ is, as under the random graph model [24], no greater than logarithmic, and (2) the scaling of clustering coefficient $C$ exceeds the scaling, $C \sim 1 / N$, expected under the random graph model [15]. 
The scale-free property drastically impacts dynamical processes on networks [25, 26]. The scale-free property is defined in terms of degree $d$, which is the number of neighbours a node possesses. For the undirected networks without self-loops or duplicate edges which we consider, the mean degree is twice the ratio $m$ of the total number of edges to the total number of nodes. For the network growth models which we examine, this ratio $m$ equals the number of new links which each incoming node distributes. The scale-free property is satisfied when, in the limit of large network size $N$, degree distribution $P(d)$ follows a power law $P(d) \propto d^{-\gamma}$ in the limit of high degree d. [27] We investigate this property using the Kolmogorov-Smirnov distance $D_{\mathrm{KS}}$ between the empirical distribution of degrees and a particular distribution which follows a power law $P(d) \propto d^{-\gamma}$ in the limit of high degree $d$ (Section 3).

It is important to capture the scale-free and small world properties because they are observed in many real-world social networks [15]. Properties which can be desirable in social networks are efficiency, unity and robustness. Network efficiency $E$ is the average rate at which information passes between distinct nodes in the network, assuming that the information travels at a rate of one link per time step [28]. Network efficiency can be expressed

$$
E=\frac{1}{N(N-1)} \sum_{i=1}^{N} \sum_{\substack{j=1 \\ j \neq i}}^{N} \rho_{i, j}{ }^{-1} .
$$

Unity can be thought of as the absence of sectarianism, which we will assume is reflected in community structure. Community structure is said to exist when the nodes of a network can be partitioned into classes such that they are significantly more likely to connect to nodes which share their class than with those from a different class. The community structure evident from a partition $c$ can be measured via its modularity [29, 30, 21]

$$
Q_{c} \triangleq \frac{1}{D} \sum_{i=1}^{N} \sum_{j=1}^{N}\left(a_{i j}-\frac{d_{i} d_{j}}{D}\right) \delta_{c(i) c(j)}
$$

where $d_{i}$ denotes the degree of node $i, D=\sum_{i=1}^{N} d_{i}$ is the sum of all degrees, and $\delta_{c(i) c(j)}$ is unity when nodes $i$ and $j$ have been partitioned into the same class, but is zero otherwise. A higher modularity $Q_{c}$ suggests that the partition $c$ 
better reflects community structure. The modularity $Q$ of a network refers to the maximum over all possible partitions $c$ of the modularity $Q_{c}$ with respect to a particular partition $c$ or, at least, the maximum modularity over all partitions which are considered. We use a fast greedy mono-scale modularity maximisation method [31] to identify a partition $c$ whereby to estimate the modularity $Q$ of a network. We assume that more unified societies separate into communities less cleanly and so exhibit lower values of the modularity $Q$.

Network robustness is assessed based on the change of size of the largest connected component, which is the largest connected subgraph of a network [15, 32]. Specifically, the robustness of a network is the fraction of nodes it can lose before collapsing into fragments [15], and depends upon whether nodes are deleted at random or in a targeted manner. Robustness to failures $f_{F}$ is the average fraction of nodes which can randomly be removed from a network before the network's largest connected component reaches less than $5 \%$ of its initial size. Robustness to attacks $f_{A}$ is the analogous fraction in which, instead of nodes randomly being removed, in each removal step the highest degree node of the network is determined and removed. Of course, other attack strategies are possible: nodes can be chosen on the basis of measures other than degree, such as betweenness centrality, eigenvector centrality, closeness centrality [33], the number of nodes at the end of walks of length two [34], coreness [35] or the number of smallest cycles in which a node participates [36]. In another variant, the ranking can be fixed according to the centrality scores of nodes in the original graph, in a simultaneous attack, instead of, as in the sequential attacks which we consider, recalculating centrality after each node removal [33, 37].

A network is called bipartite when its collection of nodes can be partitioned into two sets $U$ and $V$ such that each edge is between a node in $U$ and a node in $V$. The bipartite property is of interest because, at least up to an approximation, it is possessed by important social networks, such as teacherstudent relationships and heterosexual romantic relationships between men and women. A complete bipartite graph on $s$ and $t$ nodes is a network which can be partitioned into a set $U$ containing $s$ nodes and a set $V$ containing $t$ nodes such that each edge is between a node in $U$ and a node in $V$, and each node in $U$ is connected to each node in $V$.

We also consider the mean squared degree, $\left\langle d^{2}\right\rangle=\frac{1}{N} \sum_{i=1}^{N} d_{i}{ }^{2}$, for the pragmatic reason that ranking real-world networks by this statistic makes plots 
of network properties easier to interpret (Section 5).

\section{Modelling inclusivity and exclusivity}

In this section we propose a network growth model and rewiring algorithm to investigate the impact of inclusivity and exclusivity. In addition, we demonstrate a few properties of the proposed network growth model.

Consider an undirected network, without self-loops, which grows by the sequential addition of nodes from an initial network of $m_{0}$ nodes. As each new node enters, it links to $m \leq m_{0}$ established nodes. Under preferential attachment, the probability that an established node receives a link of an incoming node is proportional to the degree of the established node. That is, for $i=1,2, \ldots, m$, the probability $p^{[i]}(j)$ that node $j$ receives link number $i$ of the incoming node is

$$
p^{[i]}(j)=\frac{d_{j}}{D}
$$

As a network grows under preferential attachment the distribution of its degree $d$ approaches [38, 39, 40], for $d \geq m$,

$$
P(d)=\frac{2 m(m+1)}{d(d+1)(d+2)}
$$

As degree $d$ grows large, the right hand side is $\sim 2 m(m+1) d^{-3}$. Hence, preferential attachment generates a scale-free network with scale exponent $\gamma=3$.

More realistically, the number $m$ of links which each incoming node distributes as it enters might vary as the network evolves. Approximating it as a constant allows us to condition on mean degree and limiting degree distribution.

\subsection{The Barabási-Albert model}

The most popular model for growth under preferential attachment is the Barabási-Albert (BA) preferential attachment model [41]. Under BA, each of the $m$ neighbours of an incoming node is chosen sequentially, with probability proportional to degree. However, if this would result in a duplicate link (that is, if the node tentatively chosen to receive link $i$ of the incoming node is the same as the node already chosen to receive any of the links numbered 
$1,2, \ldots, i-1)$ then selection of node $i$ is repeated until the resulting choice for neighbour $i$ would not result in a repeated link.

The BA model can be thought of as inclusive because each target of a link of an incoming node can be arbitrarily far from the collection of existing contacts. In other words, under the BA model, large separations in a social network pose no obstacle to the formation of new relationships. In the next section we define a model with an upper limit to the distance between distinct targets of links of an incoming node.

\subsection{Definition of the inclusivity model}

The inclusivity model has a positive integer-valued inclusivity parameter $r$ which corresponds to the maximum allowable separation between a new contact and the set of established contacts. Thus the inclusivity $r$ represents the maximum amount of novelty or "otherness" which either incoming or established nodes can tolerate in new contacts.

To generate a network of $N$ nodes with mean degree $2 m$, the inclusivity model with inclusivity parameter $r<\infty$, which we will also refer to as the I- $r$ model, starts with a simple, connected network comprising $m_{0} \geq$ $2 m+1$ nodes, labelled $t=1,2, \ldots, m_{0}$, and $m m_{0}$ links. For each time $t=m_{0}+1, m_{0}+2, \ldots, N$ :

1 Add node $t$ to the network and link it to a single established node with probability proportional to degree (according to Eq. (1)).

2 For link number $i=2,3, \ldots, m$ :

a Perform a random walk of length $r+1$ which starts at node $t$ but does not return to node $t$ at any other point (The first step of this random walk traverses one of the $i$ established links of the incoming node, chosen uniformly at random). Let $j$ be the node at which the random walk ends.

$\mathrm{b}$ If there is already a link between $t$ and node $j$ then return to Step 2a. Otherwise, link node $t$ and node $j$.

Figure 1 sketches the process whereby an incoming node forms new connections under the inclusivity model.

We define the inclusivity model with inclusivity parameter $r=\infty$, which we will also refer to as the I- $r$ model, to coincide with the BA model. This definition is motivated by the fact that, on an undirected connected graph 


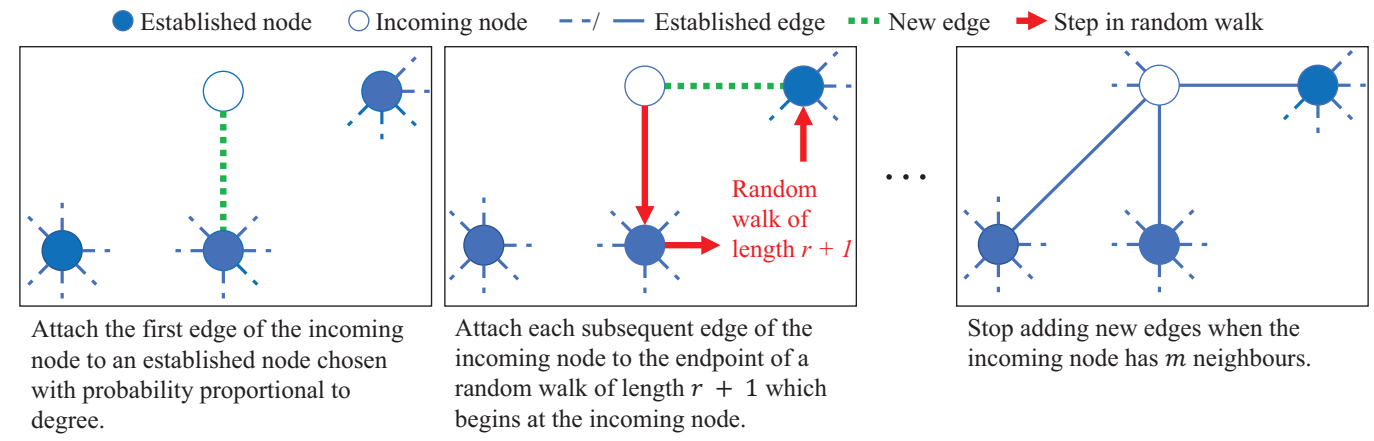

Figure 1: Under the inclusivity model, each node forms $m$ connections as it enters (For full details, please see Section 3.2 .

which does not happen to be bipartite, the end point of a sufficiently long random walk must be distributed according to the preferential attachment probability distribution Eq. (1) [42].

\subsection{Properties of the inclusivity model}

In this section we show that the inclusivity model satisfies preferential attachment and, in special cases of the model, bound the scaling with network size of the clustering coefficient.

\subsubsection{Preferential attachment}

Neglecting issues associated with duplicate edges and self-loops, for arbitrary positive integer $r$ the I- $r$ model satisfies preferential attachment. For $i>1$, the probability that node $j_{i}$ is chosen to receive link $i$ of an incoming node is

$$
p^{[i]}\left(j_{i}\right)=\sum_{k=1}^{i-1} \frac{1}{i-1} \sum_{j=1}^{N} q_{r}\left(j_{i} \mid j\right) p^{[k]}(j),
$$

where $p^{[1]}(j)$ denotes the probability that node $j$ is chosen to receive the first link and $q_{r}\left(j_{i} \mid j\right)$ is the probability that a random walk of length $r$ which begins at node $j$ will end at node $j_{i}$. Since each random walk of length $r \geq 1$ comprises a random walk of length 1 followed by another of length $r-1$, by inductively assuming that $p^{[k]}(j)=\frac{d_{j}}{D}$, the inner sum on the right hand side 
of Eq. (3) can be written

$$
\begin{aligned}
\sum_{j=1}^{N} q_{r}\left(j_{i} \mid j\right) p^{[k]}(j) & =\sum_{j=1}^{N} \sum_{l=1}^{N} q_{r-1}\left(j_{i} \mid l\right) q_{1}(l \mid j) \frac{d_{j}}{D}, \\
& =\sum_{j=1}^{N} \sum_{l=1}^{N} q_{r-1}\left(j_{i} \mid l\right) \frac{a_{j l} l}{d_{j}} \frac{d_{j}}{D}, \\
& =\sum_{l=1}^{N} q_{r-1}\left(j_{i} \mid l\right) \frac{d_{l}}{D} .
\end{aligned}
$$

Iterating this step we reach

$$
\begin{aligned}
\sum_{j=1}^{N} q_{r}\left(j_{i} \mid j\right) p^{[k]}(j) & =\sum_{h=1}^{N} q_{1}\left(j_{i} \mid h\right) \frac{d_{h}}{D} \\
& =\sum_{h=1}^{N} \frac{a_{h j_{i}}}{d_{h}} \frac{d_{h}}{D}=\frac{d_{j_{i}}}{D},
\end{aligned}
$$

which, with Eq. (3), gives the preferential attachment rule Eq. (1).

Preferential attachment is important because it implies that the large network size degree distribution under the I- $r$ model follows Eq. (2). In this way, the inclusivity model can be used to vary network properties while fixing, and thus factoring out, the role of limiting degree distribution. We emphasise that the scale-free property depends, not on our consideration of inclusivity, but upon the preferential attachment property of the inclusivity model. That is, the same scale-free limiting degree distribution is achieved with the minimally inclusive I-1 model, the maximally inclusive I- $\infty$ (or, equivalently, BA) model, and for any intermediate value of the inclusivity $r$.

\subsubsection{Clustering coefficient}

We derive theoretical results for the scaling of the clustering coefficient in two special situations. The first case involves inclusivity parameter $r=1$, and the second involves even values of the inclusivity parameter $r$.

Unsurprisingly, for $m>1$ the I-1 model leads to networks with higher clustering coefficient $C$ than does the BA model with the same number $m$ of links per incoming node. Barabási and Pósfai [15] estimated that, under BA, for $m>1$ the clustering coefficient scales with network size $N$ as 
$\frac{1}{N}(\log N)^{2}$. In contrast, under I-1 the global clustering coefficient should scale as $(\log N)^{-1}$ or more. To see this, note that Barabási and Pósfai [15] estimate the degree of node $i$ of a network growing under preferential attachment to be $d_{i}=m \sqrt{N / i}$. It follows that the number of pairs of adjacent edges can be estimated to scale as $\sum_{i=1}^{N} \frac{1}{2} d_{i}\left(d_{i}-1\right) \sim N \log N$. Also, under I-1, the number of triangles increases by at least $m-1$ each time a node enters. Hence we can estimate that when $m>1$ the global clustering coefficient $C$, which is three times the ratio of the number of triangles to the number of pairs of adjacent edges [15], scales as at least $N /(N \log N)=(\log N)^{-1}$.

In the preceding paragraph it was shown that, for $m>1$, the scaling with network size of the clustering coefficient under the inclusivity model is larger when $r=1$ than when $r=\infty$. It might be reasonable to suppose that clustering coefficient will decrease monotonically with increasing inclusivity $r$, but we can show that this is not necessarily the case. When inclusivity $r$ is odd we would expect some triangles and so a non-zero value of the clustering coefficient $C$, but when $r$ is even certain initial conditions forbid the formation of triangles. Indeed, the inclusivity model can describe the growth of bipartite networks, and a graph is bipartite if and only if it has no odd cycles [43], and in particular no triangles.

To see that when the inclusity $r$ is even the inclusivity model can preserve the bipartite property, and hence can generate networks with vanishing clustering coefficient, consider a node entering a bipartite network under the inclusivity model with even inclusivity $r$. Assume that, before the node enters, the nodes of the graph can be divided into disjoint sets $U$ and $V$ such that each edge in the graph joins a node in $U$ and one in $V$. Without loss of generality, assume that the recipient of the first link of an incoming node $i$ lies in $U$. We will show that after node $i$ enters the new graph will be able to be divided into disjoint sets $U$ and $V \cup\{i\}$ such that each edge in the graph joins a node in $U$ and one in $V \cup\{i\}$. This will show that, after an iteration of the model, the graph is still bipartite. Let integer $k$ be 2 or greater and assume inductively that, for $i=1, \ldots, k-1$, the target of neighbour $i$ of the incoming node lies in $U$. The endpoint of a random walk of length $r+1$ which begins at the incoming node will be distributed as the endpoint of a random walk of length $r$ which begins at an immediate neighbour of the incoming node, and all of these neighbours belong to the set $U$. Since the graph is bipartite the endpoint of this random walk of even length must end 
at an element of the set $U$.

When the initial graph is bipartite, the clustering coefficient remains fixed as the even-valued inclusivity $r$ varies. In this way, the inclusivity model can fix and thus factor out the role of clustering coefficient while allowing other properties to vary.

\subsection{Inclusive and exclusive rewiring}

Exclusive (inclusive) rewiring is intended to emulate the I- $r$ (I- $\infty$ ) model while randomising connections and maintaining the degree distribution to the extent which is convenient without incurring self- or duplicate links. A simple network with at least one edge undergoes exclusive rewiring with inclusivity $r$ by first severing all links between nodes so that each node has a number of stubs equal to its degree in the original network. Subsequently:

1 Choose a stub uniformly at random and let $i$ be the node to which the stub belongs.

2 a Let a walk which begins at node $i$ and does not return to node $i$ be called a relevant walk. If there is a node with a stub which is not a neighbour of node $i$ and lies at the end of a relevant walk of length $r+1$ then choose such a node $j$ with probability proportional to the number of relevant walks of length $r+1$ which end in node $j$.

b Otherwise, if there are stubs attached to nodes which are a distance greater than or equal to 2 from node $i$ then choose such a stub uniformly at random. Let $j$ be the node to which this stub belongs.

c Otherwise, do not choose a node $j$.

3 If nodes $i$ and $j$ were chosen then add an edge between these nodes and remove a stub from each.

4 a If there exists a pair of stubs which belong to distinct, non-neighbouring nodes then return to Step 1.

b Otherwise, remove all remaining stubs, which cannot be combined into edges without incurring self- or duplicate links, and end the rewiring process. 
Figure 2 illustrates two stubs combining to form an edge under exclusive rewiring with inclusivity parameter $r$. A simple network with at least one edge undergoes inclusive rewiring in the same manner, except that Step 2a is omitted. Instead, Step $2 \mathrm{~b}$ proceeds directly, as though the condition of Step 2a could not be satisfied. Inclusive rewiring only differs from employing the stub configuration model [44] in that inclusive rewiring avoids self- and duplicate links and may not exactly preserve the degree sequence.

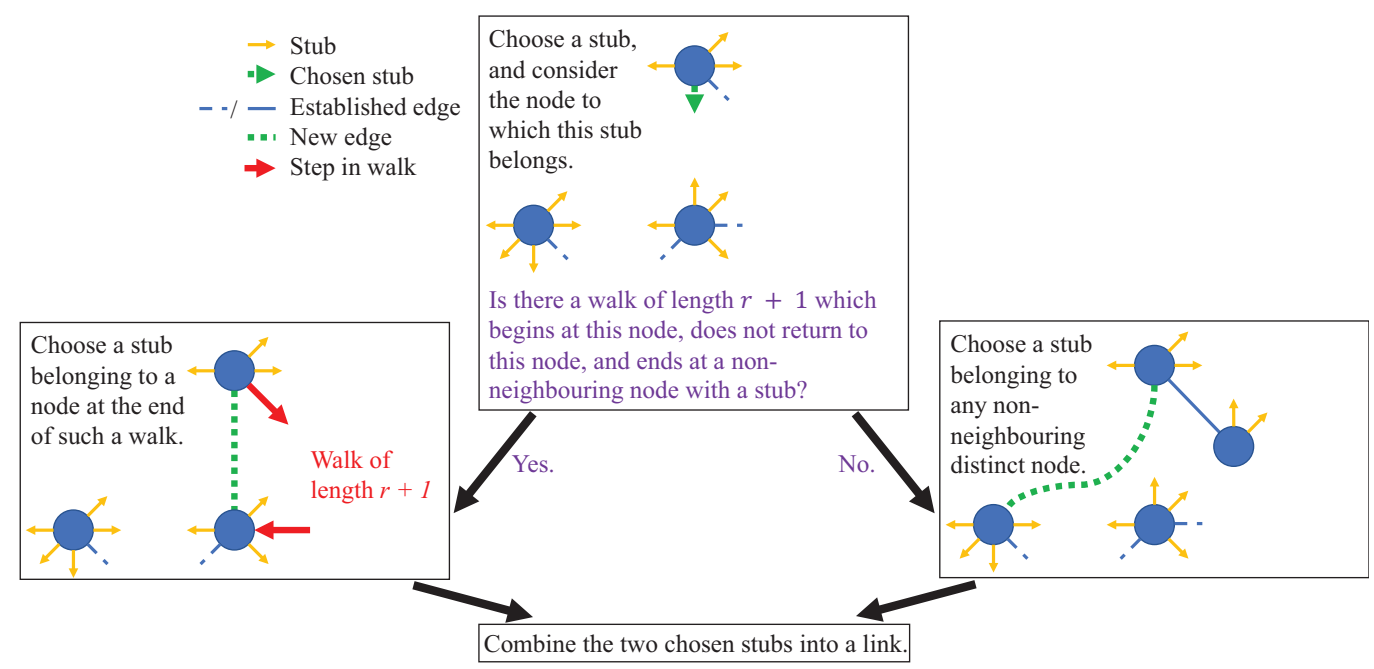

Figure 2: Exclusive rewiring with inclusivity $r+1$ favours the connection of nodes which are at opposite ends of a random walk of length $r+1$ (For full details, please see Section 3.4 .

\section{Methods}

The effect of inclusivity $r$ on growing networks is assessed by growing networks with $m=1,2, \ldots, 8$ new links per incoming node starting from, except where noted otherwise, the complete graph on $2 m+1$ vertices. This graph is the smallest simple graph which allows the mean degree of the growing network to remain constant. Statistics are calculated on each growing network when it has $N=32,64,128, \ldots, 8192$ nodes.

The real-world importance of forming relationships inclusively is explored by inclusive and exclusive rewiring (Section 3.4) of twelve social networks. In Table 1 are listed the number $N$ of nodes, and ratio $m$ of links to nodes, and the mean squared degree $\left\langle d^{2}\right\rangle$ of each of these social networks. 
Table 1: Basic properties of twelve real-world social networks, ordered by mean squared degree $\left\langle d^{2}\right\rangle$. Each network with name beginning "HS" and full name beginning "COMM" comprises the largest connected component of a school friendship network, inferred as detailed at http://moreno.ss.uci.edu/data.html\#adhealth. These six datasets were chosen uniformly at random, without replacement, from among those for which the largest connected component of the first school of the dataset contained at least 500 nodes. Directions, weights and multiplicity of links were ignored. Each network with full name beginning "soc-" is the largest connected component of a network obtained from the Network Data Repository 45, at http://networkrepository.com/soc.php.

\begin{tabular}{llccc} 
Name & Full name & $N$ & $m$ & $\left\langle d^{2}\right\rangle$ \\
\hline HS 1 & COMM_84 & 722 & 2.95 & 46.6 \\
HS 2 & COMM_81 & 1290 & 2.94 & 46.6 \\
HS 3 & COMM_25 & 790 & 3.25 & 54.9 \\
HS 4 & COMM_79 & 1190 & 3.49 & 62.3 \\
HS 5 & COMM_26 & 551 & 3.75 & 71.9 \\
HS 6 & COMM_50 & 630 & 4.03 & 86.1 \\
Vote & soc-wiki-Vote & 889 & 3.28 & 119 \\
Food & soc-fb-pages-food & 620 & 3.37 & 134 \\
Show & soc-fb-pages-tvshow & 3892 & 4.43 & 236 \\
Advo. & soc-advogato & 5908 & 7.06 & 603 \\
Hams. & soc-hamsterster & 2000 & 8.05 & 704 \\
Poli. & soc-fb-pages-politician & 5054 & 7.79 & 1290
\end{tabular}

\section{Results and discussion}

Figure 3(a) shows that the I-1 and I-3 network growth models leads to degree distributions which fit the limiting distribution (2) about as well as those provided by the Barabási-Albert (BA) (or, equivalently, the I- $\infty$ ) model. The same is true for other values of inclusivity $r$, meaning that the inclusivity model retains the scaling properties for which the BA (or I- $\infty$ ) model is celebrated. Figure 3(b) shows that, under the inclusivity model with $r=1$ or $r=3$, mean distance $\langle\rho\rangle$ scales with network size $N$ at most as $\langle\rho\rangle \sim \log N$. Once again, the same is true for other values of inclusivity $r$, meaning that the inclusivity model exhibits the internode separation properties necessary to obtain the small world property.

Figure 4 presents trends in the clustering coefficient $C$ under the inclusivity model. Figure 4(a) supports the idea that under I-1, but not under either I-3 with $m=6$ new links per incoming nodes or I- $\infty, C^{-1}$ is approximately affine in $\log N$. Figure 4(b) supports the result (Section 3.3.2) that under the 


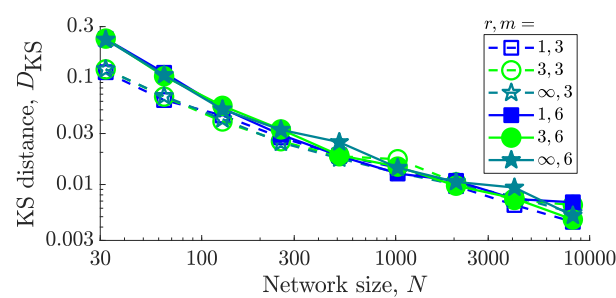

(a)

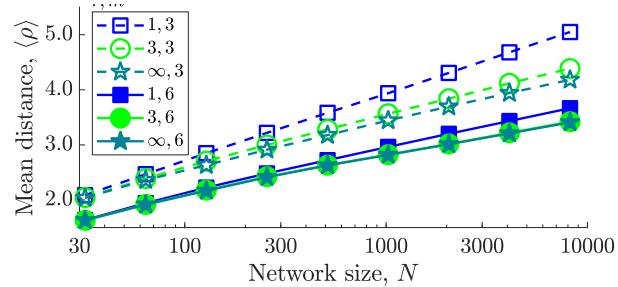

(b)

Figure 3: Variation with network size $N$ of (a) the Kolmogorov-Smirnov distance $D_{\mathrm{KS}}$ between the empirical degree distribution and the limiting distribution Eq. (2) and (b) the mean internode distance $\langle\rho\rangle$, for networks grown under the I- $r$ model. The inclusivity model with inclusivity $r=1$ or $r=3$ leads to degree distributions which fit the limiting distribution Eq. (2) as closely as those provided by the inclusive Barabási-Albert model (or, equivalently, the inclusivity model with inclusivity $r=\infty$ ), and in each of these cases the mean internode distance $\langle\rho\rangle$ scales with network size $N$ at most as $\langle\rho\rangle \sim \log N$. The markers show the mean over 10 trials; the standard deviation over these trials was smaller than the extent of the markers.

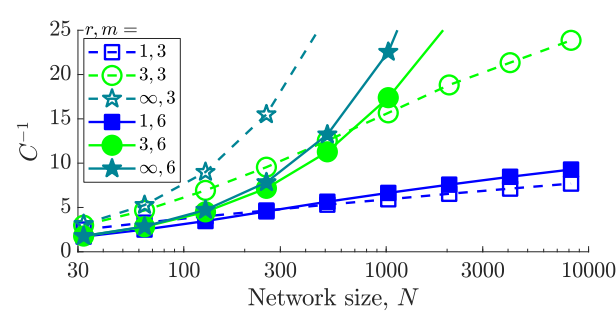

(a)

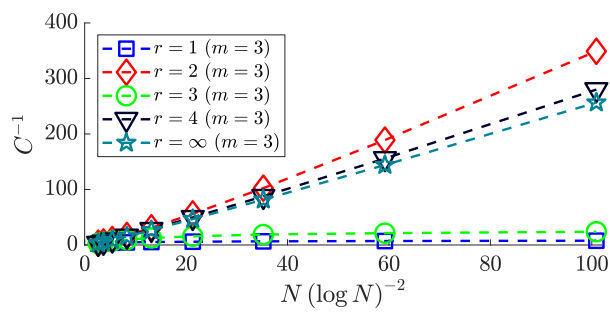

(b)

Figure 4: Variation of the inverse $C^{-1}$ of the global clustering coefficient $C$ with (a) network size $N$, and (b) $N(\log (N))^{-2}$, for networks grown under the I- $r$ model. When $r=1$, but not when $r=\infty, C^{-1}$ is approximately affine in $\log N$. When inclusivity $r$ is even or $r=\infty$, for the initial conditions of this figure it appears that $C^{-1} \sim N(\log (N))^{-2}$. The logarithm is base $e$. The markers show the mean over 10 trials.

inclusivity model with inclusivity $r=\infty, C^{-1}$ scales as $C^{-1} \sim N(\log (N))^{-2}$. This figure also suggests that, for other values of the inclusivity and with the initial condition of a complete graph, the scaling of the clustering coefficient $C$ is $\sim N^{-1}(\log (N))^{2}$ or greater. The clustering coefficient under the random graph model scales [15] as $C \sim 1 / N$ so, for large network size, would be expected to be lower than that achieved under the inclusivity model grown from a complete graph. Hence, with a complete graph as the initial condition, 
the inclusivity model is small world.

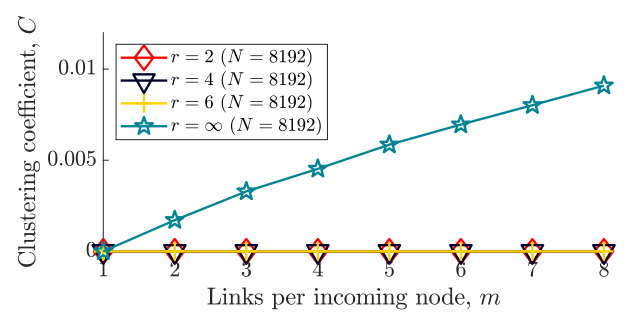

(a)

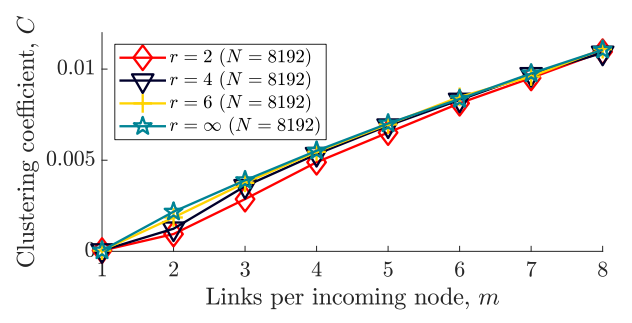

(b)

Figure 5: Variation with number $m$ of links per incoming node of the global clustering coefficient $C$ for I- $r$ networks of size $N=8192$ (a) grown from a complete bipartite graph on $2 m$ and $2 m$ nodes, and (b) grown from a complete graph on $2 m+1$ nodes. For even values of inclusivity $r$ the global clustering coefficient $C$ is, in case (a), zero, and, in case (b), of the same order of magnitude as under the Barabási-Albert model (or, equivalently, the inclusivity model with inclusivity $r=\infty$ ). The markers show the mean over 10 trials; the standard deviation over these trials was smaller than the extent of the markers.

Figure 5 shows that not all initial conditions and values of inclusivity $r$ allow the inclusivity model to provide small world networks. As Figure 5(a) illustrates, when $r$ is even and the initial graph is bipartite the clustering coefficient $C$ remains fixed at zero, meaning that the resulting graph cannot be called small world. Comparison of Figures 4 and 5(b) reveals that distinct differences between the odd $r$ case and the even $r$ case occur even for nonbipartite graphs. Specifically, for even inclusivity $r$ the clustering coefficient $C$ is less than or equal to that observed under the BA model, while for odd inclusivity $r$ it is greater. Subsequent numerical results concentrate on the odd $r$ case, which Figures $3(\mathrm{~b})$ and 4 suggest leads to graphs with the small world property.

Figure 6(a) reveals that, when the number $m$ of links per incoming node is greater than one but not too large, greater inclusivity $r$ leads to a network of higher efficiency $E$. Similarly, Figure 6(b) shows that, for moderate $m>1$, modularity $Q$ decreases as inclusivity $r$ increases. However, as the mean number $m$ of links per node increases, for $m>1$, differences in efficiency $E$ and modularity $Q$ due to differences in inclusivity $r$ lessen. Also, except when the number $m$ of links per incoming node is small, networks with any level of inclusivity $r$ greater than the most exclusive case achieve efficiencies and modularities similar to those achieved under maximal inclusivity. In all, Figure 6 suggests that organisations seeking a more efficient 


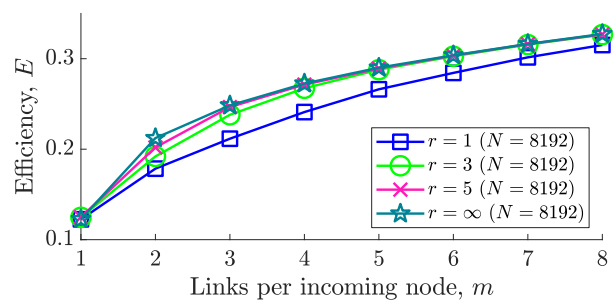

(a)

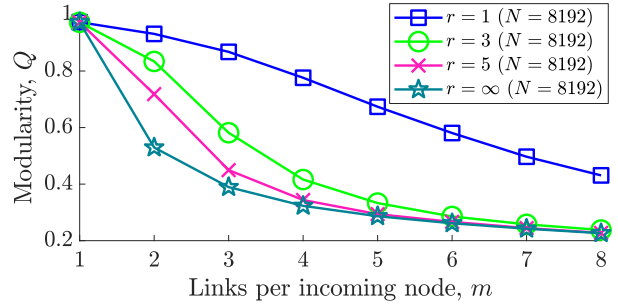

(b)

Figure 6: Variation with number $m$ of links per incoming node of (a) network efficiency $E$, and (b) modularity $Q$, for networks grown under the I- $r$ model. As inclusivity $r$ increases, the network efficiency $E$ and modularity $Q$ increase and decrease respectively. The markers show the mean over 10 trials; the standard deviation over these trials was smaller than the extent of the markers.

and unified structure should discourage exclusive formation of relationships, and encourage the formation of more relationships in general.

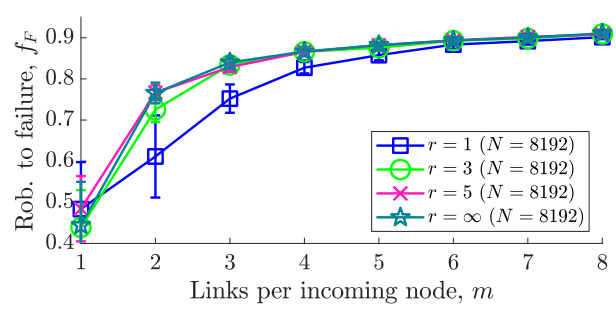

(a)

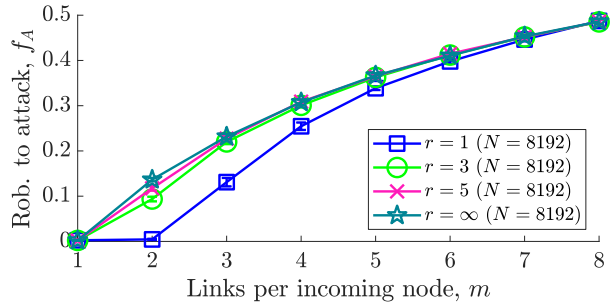

(b)

Figure 7: Variation with network size $N$ of (a) robustness to failure $f_{F}$ and (b) robustness to attack $f_{A}$, for networks grown under the I- $r$ model. Robustnesses $f_{F}$ and $f_{A}$ increase with inclusivity $r$. Markers and error bars show the mean and sample standard deviation over 10 trials.

Figure 7 suggest that, for moderate $m>1$, more inclusive networks are more robust. An increase with inclusivity $r$ of the robustness $f_{F}$ to random failure is evident, but the increase with inclusivity $r$ of the robustness $f_{A}$ to targeted attacks is even more noticeable. However, differences in robustness due to differences in inclusivity $r$ lessen as the number $m$ of links per incoming node increases, at least for $m>1$. Also, for a fixed number $m$ of links per incoming node, levels of robustness vary little as inclusivity increases beyond the minimum inclusivity, $r=1$. The results suggest that leaders wishing 
to avoid fragile societies or organisations should foster connections between diverse entities, as well as the formation of more connections in general. The error bars of Fig. 7(a) show that the standard deviation in the robustness to failure $f_{R}$ varies substantially with $r$ and $m$. This variability in robustness is explicated by comparison with Fig. 6(b), which shows the modularity $Q$. The variance in the robustness to failure $f_{F}$ increases with modularity $Q$, showing that more modular networks fragment less predictably under random deletion of nodes.

Figures 6 and 7 reveal that, except when the number $m$ of links per incoming node is small, levels of inclusivity larger than one behave similarly to the maximally inclusive case. This suggests that the presence of even a small amount of inclusivity can have a considerable influence on network properties. The extreme case with inclusivity $r=1$ exhibits the most substantial difference from the maximally inclusive $r=\infty$ case, but might not realistically represent the formation of social networks. On the other hand, this maximally exclusive case might be the most intuitive form of the inclusivity model, because it arises from the widely studied mechanism of triadic closure [13] the idea that the contact of an established contact can become a new contact.

Figure 8 suggests that the exclusive and inclusive rewiring algorithms are fulfilling the tasks for which they were designed. Figure 8(c) reveals that, even before rewiring, social divisions exist at the school. Figures 8(a) and 8(b) suggests that exclusive rewiring with lower inclusivity $r$ leads to more social modules, which are more starkly separated, while Fig. 8(d) shows that inclusive rewiring integrates all nodes into a cohesive whole.

Figures 9 (a-d) presents pertinent network properties - efficiency $E$, modularity $Q$, robustness to failure $f_{F}$ and robustness to attack $f_{A}$ - of the connected components of twelve real world networks (Table 1). The figure juxtaposes these properties with those of the connected components of the networks after exclusive or inclusive rewiring (Section 4). In all cases, the mean efficiency $E$, robustness to failure $f_{F}$ and robustness to attack $f_{A}$ are higher under inclusive random restructuring than exclusive random restructuring, while modularity $Q$ is lower. Similarly, the mean efficiency $E$, robustness to failure $f_{F}$ and robustness to attack $f_{A}$ (the mean modularity $Q$ ) are higher (lower) under exclusive random restructuring with inclusivity parameter $r=2$ than with inclusivity parameter $r=1$, with a single exception provided by the mean efficiency $E$ of the exclusively restructured network "Poli". This supports the idea that inclusivity provides benefits in terms of 


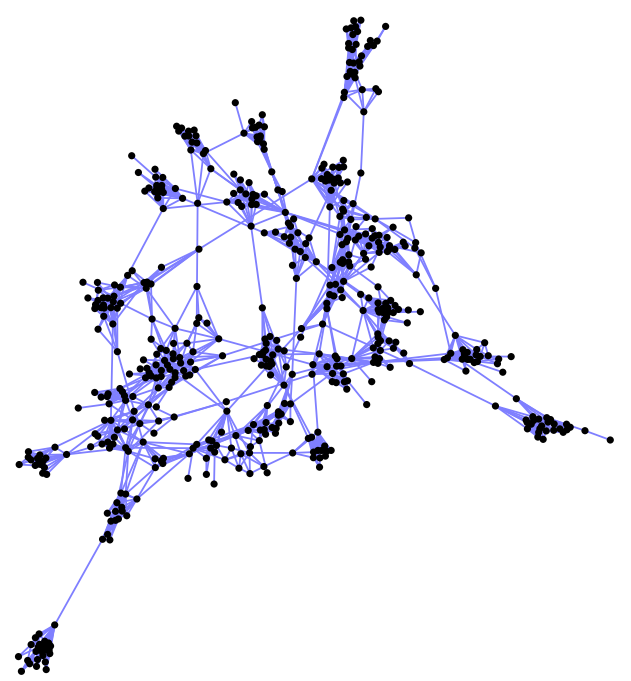

(a) Exclusive $(r=1)$

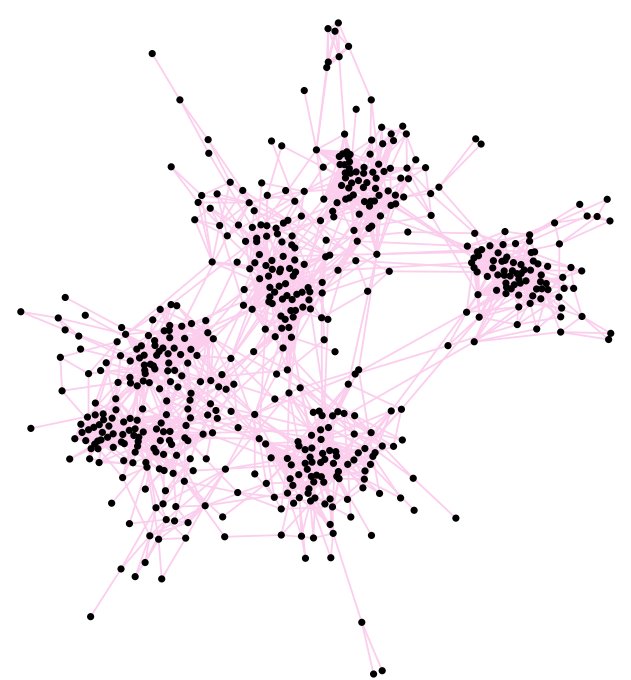

(c) Original

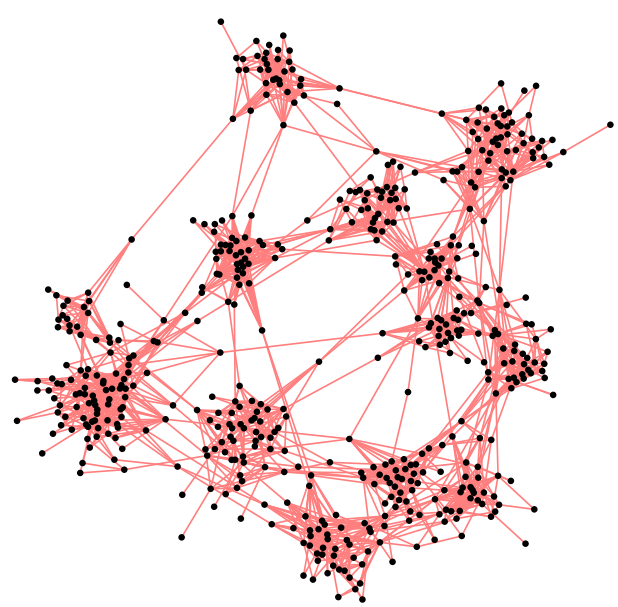

(b) Exclusive $(r=2)$

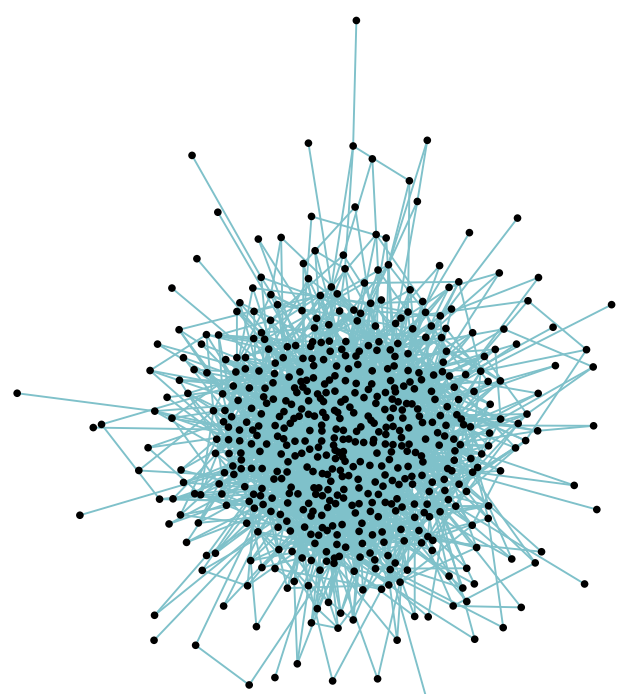

(d) Inclusive ${ }^{\cdot}$

Figure 8: Force-directed layout [46] of the high school friendship network HS 1 (Table 11) (a) after exclusive rewiring with inclusivity $r=1$, (b) after exclusive rewiring with inclusivity $r=2$, (c) in its original form, and (d) after inclusive rewiring. Exclusive rewiring makes this high school's already modular social network even more schismatic, while inclusive rewiring blurs social distinctions.

efficiency, unity and robustness. However, it is not universally the case that inclusive (exclusive rewiring with inclusivity $r=1$ ) rewiring increases (de- 


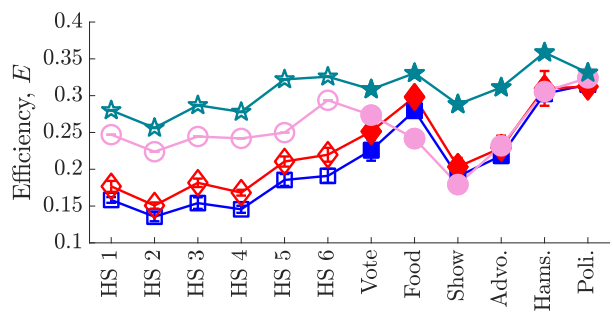

(a)

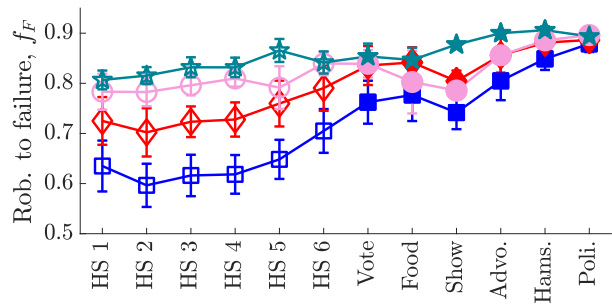

(c)

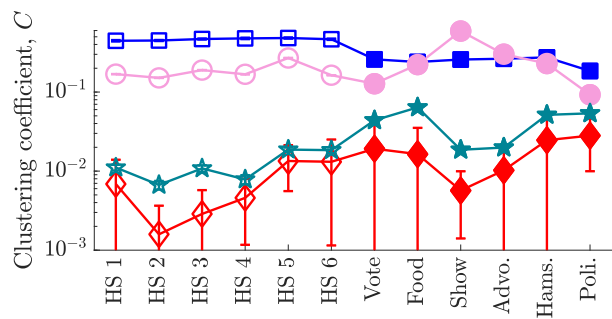

(e)

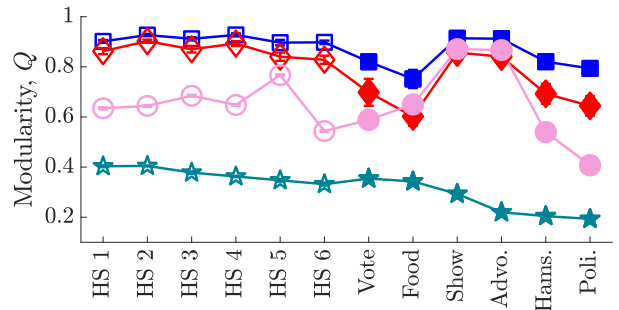

(b)

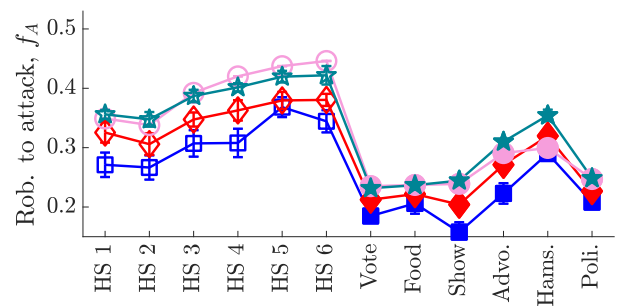

(d)

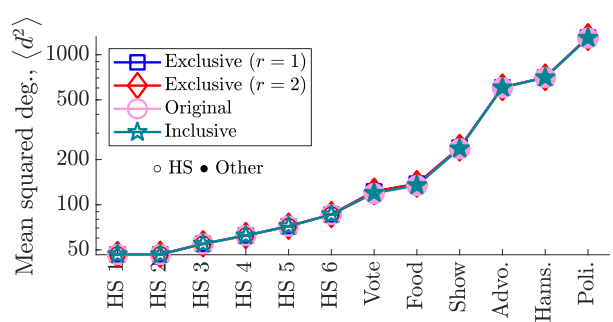

(f)

Figure 9: (a) Efficiency $E$, (b) modularity $Q$, (c) robustness to failure $f_{F}$, (d) robustness to attack $f_{A}$, (e) global clustering coefficient $C$ and (f) mean squared degree $\left\langle d^{2}\right\rangle$ of the connected components of twelve social networks and the connected components of versions of these networks randomly rewired in an inclusive way or in an exclusive way with inclusivity parameter either $r=1$ or $r=2$. Inclusive rewiring leads to higher efficiency and robustness and lower modularity than does exclusive rewiring. Markers and error bars show the mean and sample standard deviation over 10 trials.

creases) efficiency and robustness, and decreases (increases) modularity with respect to the values in the original social network. In two cases, "Food" and "Show", the mean efficiency $E$ after exclusive rewiring with inclusivity $r=1$ is higher than in the original network. In five other cases, "HS 3""HS 6" and "Vote", mean robustness to attack after inclusive restructuring 
is lower than for the original network. The unexpected direction of these changes may reflect the fact that these social networks actually arose via a complicated and unknown process, and inclusive (exclusive) restructuring is unlikely to achieve exactly the same topology as would growing the original networks in a more inclusive (exclusive) manner. When the ambiguities of the real-world network growth process are factored out by restructuring at random, exclusivity - as represented by the exclusive rewiring algorithm leads to consistently lower mean efficiency and robustness, and consistently higher mean modularity, than inclusivity. In sum, Figure 9(a-d) suggests that shifts towards greater exclusivity will likely lead to worse outcomes in terms of efficiency, unity and robustness than shifts towards greater inclusivity.

Analogously, as Figure 9(e) shows, in all cases the clustering coefficient $C$ is lower under inclusive restructuring than exclusive restructuring with inclusivity $r=1$, and is lowest under exclusive restructuring with inclusivity $r=2$. Assigning links between the end points of random walks of length two discourages the formation of triangles. However, not in every case does exclusive rewiring with inclusivity $r=1$ increase the clustering coefficient: the mean clustering coefficient of "Show" and "Advo." is lower after exclusive rewiring with inclusivity $r=1$. The sample standard deviation of the clustering coefficient $C$ is always small (less than 0.03 ) in absolute value but, in the case of exclusive rewiring with inclusivity $r=2$, error bars are prominent because of the logarithmic scale of the ordinate.

Figure $9(\mathrm{f})$ shows that the mean squared degree $\left\langle d^{2}\right\rangle$ is similar before and after rewiring, a consequence of the fact that the rewiring algorithms preserve degree sequence as much as is convenient without incurring self- or duplicate links (Section 3.4). Networks are arranged by the mean squared degree $\left\langle d^{2}\right\rangle$ because this choice lends order to Fig. 9. This ordering makes adjacent the two cases in which exclusive rewiring with inclusivity $r=1$ increases network efficiency, the two cases in which mean clustering coefficient is lower after exclusive rewiring with inclusivity $r=1$, as well as the five cases in which inclusive restructuring reduces the mean robustness to targeted attack, and also positions at a boundary the case for which the efficiency $E$ is higher under exclusive rewiring with inclusivity parameter $r=1$ than with inclusivity parameter $r=2$. Figures 9(a) and 9(c) suggest that the differences in the efficiencies $E$ and robustnesses to random failure $f_{F}$ of inclusively and exclusively rewired networks tend to decrease as mean squared degree $\left\langle d^{2}\right\rangle$ increases. In contrast, as Fig. 9(b) and 9(d) show, in terms of modularity $Q$ and robustness to targeted attack $f_{A}$, the difference between inclusively and 
exclusively rewired networks varies little - or, at least, less systematically with mean squared degree $\left\langle d^{2}\right\rangle$.

\section{Conclusion}

We proposed a simple model geared towards gauging the role of inclusivity in a growing or restructured organisation. Under our model, the maximum separation between new and established contacts is bounded above by an integer-valued inclusivity parameter $r$, which represents the maximum amount of novelty or "otherness" which people can accept. In addition we consider the effect of randomly rewiring real-world social networks in either an exclusive or inclusive way. Our results show that choosing inclusivity over exclusivity tends to increase network efficiency, encourage unity (by reducing modularity) and increase a network's robustness to either random failure or targeted attack. However, at least for structures grown via our network growth model, these properties improve, and differences as a function of inclusivity become less pronounced, as the ratio of links to nodes increases. The analysis of network growth models also suggested that the presence of even a small amount of inclusivity is influential: except for the smallest ratios of links to nodes, networks with inclusivity higher than the minimum possible level displayed efficiency and robustness similar to those exhibited by the most inclusive networks.

The inclusivity model is based on a simple unweighted random walk mechanism which, neglecting subtleties associated with self-loops and duplicate edges, leads to degree distributions identical, in the large network size limit, to those of the extensively studied Barabási-Albert (BA) network model. Not only does this invariance allow the limiting degree distribution to be factored out of studies, it could also allow the concept of inclusivity to be incorporated into the multitude of existing numerical studies which employ BA networks. Even values of the inclusivity parameter $r$ could be used to model the growth of bipartite networks and can generate networks without any triangles and zero clustering coefficient, so that clustering coefficient could also be fixed while investigating the impact of other network characteristics. Simulations suggest that, when inclusivity $r$ is odd, networks produced under the proposed model possess the small world property.

The inclusivity model consistently leads to networks with scale exponent $\gamma=3$, but the scale exponents of real-world networks are not restricted in this way. Bianconi and Barabási [47] showed that modifying the BA network 
growth model by randomly assigning to each incoming node a fitness which weights its likelihood of receiving incoming links makes accessible a range of scale exponents. The inclusivity model could be modified in an analogous way; by assigning to each incoming node a random fitness which weights its probability of being the recipient of the first link of each subsequent incoming node and the destination of each step of a random walk. This adaptation could allow the inclusivity model to offer a spectrum of scale exponents. A more direct way simultaneously to choose inclusivity and scale exponent would be applying the inclusive rewiring model to a scale-free network with the desired scale exponent, an approach which would exploit the fact that exclusive rewiring approximately preserves degree sequences.

The links of many social networks are weighted [48, 49], and these weights have been shown to be crucial for reliably assessing network robustness 32 , 49]. The importance of link weights motivates the extension of the inclusivity and rewiring model to weighted networks. It might be feasible to extend the exclusive rewiring model in a way which approximately preserves node strength by only combining stubs into links when links from which the stubs emerged had similar weights. The inclusivity model with inclusivity parameter $r$ could be extended to weighted networks with non-negative weights via three small modifications: (1) the first recipient of a link of an incoming node would be chosen with probability proportional to node strength, which is the sum of the weights of the links in which a node participates, (2) subsequent recipients would be chosen as the endpoint of random walks of length $r+1$ which begin at the incoming node, do not return to it, and each step of which is chosen with probability proportional to link weight, and (3) new edges would be assigned weights $w$ drawn randomly from a fixed, non-negative probability distribution which decays more rapidly than $w^{-3}$. The attractions of this formulation include: (1) the expected mean node strength of the network would be fixed, and (2) the model would satisfy a version of preferential attachment in which mean degree is replaced by node strength and so should produce a node strength-based analogue of a scale-free network [50].

Since their first conception the small world and BA models have found continuous employment among researchers probing the impact of small world and scale-free properties on the spread of diseases, the propagation of information and a host of other dynamical processes. This paper presents a single step in using network science to quantify the impact which inclusivity can have on organisations and on society. There is still much to be done to 
understand the network benefits - and, possibly, detriments - of inclusivity.

\section{Acknowledgements}

We thank anonymous reviewers for valuable comments. JMM and GY are supported by National Natural Science Foundation of China (Grant No. 11875043), Shanghai Science and Technology Committee (Grant No. 18ZR1442000). MS is supported by ARC Discovery Grant DP180100718.

\section{Data statement}

The data that support the findings of this study are openly available

on the webpage of Prof. Linton C. Freeman, at http://moreno.ss.uci. edu/data.html\#adhealth, and the Network Data Repository [45], at http: //networkrepository.com/soc.php.

\section{References}

[1] R. G. Berlach, D. J. Chambers, Inclusivity imperatives and the Australian national curriculum, in: The Educational Forum, Vol. 75, Taylor \& Francis, 2010, pp. 52-65.

[2] V. C. Plaut, F. G. Garnett, L. E. Buffardi, J. Sanchez-Burks, "What about me?" Perceptions of exclusion and Whites' reactions to multiculturalism., Journal of Personality and Social Psychology 101 (2) (2011) 337.

[3] S. Jain, R. Lobo, Diversity and inclusion: A business imperative in global professional services, in: Globalization of professional services, Springer, 2012, pp. 181-187.

[4] S. Connolley, R. S. Hausstätter, Tocqueville on democracy and inclusive education: A more ardent and enduring love of equality than of liberty, European Journal of Special Needs Education 24 (3) (2009) 231-243.

[5] K. E. Whittington, Free speech and the diverse university, Fordham Law Review 87 (2018) 2453.

[6] C. McLoughlin, Inclusivity and alignment: Principles of pedagogy, task and assessment design for effective cross-cultural online learning, Distance Education 22 (1) (2001) 7-29. 
[7] P. Gurin, B. R. A. Nagda, G. E. Lopez, The benefits of diversity in education for democratic citizenship, Journal of Social Issues 60 (1) (2004) 17-34.

[8] R. Viljoen, Inclusive Organizational Transformation, Routledge, Abingdon-on-Thames, 2014.

[9] K. L. Ashcraft, S. L. Muhr, J. Rennstam, K. Sullivan, Professionalization as a branding activity: Occupational identity and the dialectic of inclusivity-exclusivity, Gender, Work \& Organization 19 (5) (2012) 467-488.

[10] A. Wittenberg-Cox, The trouble with gender targets, Harvard Business Review Digital Articles, https://hbr.org/2013/10/ the-trouble-with-gender-targets, Accessed: 2020-01-23 (16 October, 2013).

URL https://hbr .org/2013/10/the-trouble-with-gender-targets

[11] C. M. Riordan, Diversity is useless without inclusivity, Harvard Business Review Digital Articles, https://hbr.org/2014/06/ diversity-is-useless-without-inclusivity, Accessed: 2020-01-23 (5 June, 2014).

URL https://hbr.org/2014/06/diversity-is-useless-without-inclusivity

[12] L. Sherbin, R. Rashid, Diversity doesn't stick without inclusion, Harvard Business Review Digital Articles, https: //hbr.org/2017/02/diversity-doesnt-stick-without-inclusion, Accessed: 2020-01-23 (1 February, 2017).

URL https://hbr.org/2017/02/diversity-doesnt-stick-without-inclusion

[13] G. Bianconi, R. K. Darst, J. Iacovacci, S. Fortunato, Triadic closure as a basic generating mechanism of communities in complex networks, Physical Review E 90 (4) (2014) 042806.

[14] S. N. Dorogovtsev, J. F. Mendes, A. N. Samukhin, Size-dependent degree distribution of a scale-free growing network, Physical Review E 63 (6) (2001) 062101.

[15] A.-L. Barabási, M. Pósfai, Network Science, Cambridge University Press, 2016. 
[16] P. Holme, B. J. Kim, Growing scale-free networks with tunable clustering, Physical Review E 65 (2) (2002) 026107.

[17] J. Saramäki, K. Kaski, Scale-free networks generated by random walkers, Physica A: Statistical Mechanics and its Applications 341 (2004) 80-86.

[18] T. Evans, J. Saramäki, Scale-free networks from self-organization, Physical Review E 72 (2) (2005) 026138.

[19] C. Herrera, P. J. Zufiria, Generating scale-free networks with adjustable clustering coefficient via random walks, in: 2011 IEEE Network Science Workshop, IEEE, 2011, pp. 167-172.

[20] P. Li, J. Zhang, M. Small, Emergence of scaling and assortative mixing through altruism, Physica A: Statistical Mechanics and its Applications 390 (11) (2011) 2192-2197.

[21] M. E. J. Newman, Networks: An Introduction, Oxford University Press, 2010.

[22] D. J. Watts, S. H. Strogatz, Collective dynamics of 'small-world' networks, Nature 393 (6684) (1998) 440.

[23] M. Barthélémy, L. A. N. Amaral, Small-world networks: Evidence for a crossover picture, Physical Review Letters 82 (15) (1999) 3180.

[24] E. N. Gilbert, Random graphs, The Annals of Mathematical Statistics 30 (4) (1959) 1141-1144.

[25] R. Pastor-Satorras, A. Vespignani, Epidemic spreading in scale-free networks, Physical Review Letters 86 (14) (2001) 3200.

[26] A. Arenas, A. Díaz-Guilera, J. Kurths, Y. Moreno, C. Zhou, Synchronization in complex networks, Physics Reports 469 (3) (2008) 93-153.

[27] R. Van Der Hofstad, Random Graphs and Complex networks, Vol. 1, Cambridge University Press, 2016.

[28] V. Latora, M. Marchiori, Efficient behavior of small-world networks, Physical Review Letters 87 (19) (2001) 198701. 
[29] M. E. J. Newman, Mixing patterns in networks, Physical Review E 67 (2) (2003) 026126.

[30] M. E. J. Newman, M. Girvan, Finding and evaluating community structure in networks, Physical Review E 69 (2) (2004) 026113.

[31] E. Le Martelot, C. Hankin, Fast multi-scale detection of relevant communities in large-scale networks, The Computer Journal 56 (9) (2013) $1136-1150$.

[32] M. Bellingeri, D. Cassi, Robustness of weighted networks, Physica A: Statistical Mechanics and its Applications 489 (2018) 47-55.

[33] S. Iyer, T. Killingback, B. Sundaram, Z. Wang, Attack robustness and centrality of complex networks, PLoS One 8 (4) (2013) e59613.

[34] M. Bellingeri, D. Bevacqua, F. Scotognella, L. Zhe-Ming, D. Cassi, Efficacy of local attack strategies on the beijing road complex weighted network, Physica A: Statistical Mechanics and its Applications 510 (2018) 316-328.

[35] M. Kitsak, L. K. Gallos, S. Havlin, F. Liljeros, L. Muchnik, H. E. Stanley, H. A. Makse, Identification of influential spreaders in complex networks, Nature Physics (2010).

[36] T. Fan, L. Lü, D. Shi, T. Zhou, Characterizing cycle structure in complex networks, arXiv preprint arXiv:2001.08541 (2020).

[37] M. Bellingeri, D. Cassi, S. Vincenzi, Efficiency of attack strategies on complex model and real-world networks, Physica A: Statistical Mechanics and its Applications 414 (2014) $174-180$.

[38] P. L. Krapivsky, S. Redner, F. Leyvraz, Connectivity of growing random networks, Physical Review Letters 85 (21) (2000) 4629.

[39] S. N. Dorogovtsev, J. F. F. Mendes, A. N. Samukhin, Structure of growing networks with preferential linking, Physical Review Letters 85 (21) (2000) 4633.

[40] B. Bollobás, O. Riordan, J. Spencer, G. Tusnády, The degree sequence of a scale-free random graph process, Random Structures \& Algorithms 18 (3) (2001) 279-290. 
[41] A.-L. Barabási, R. Albert, Emergence of scaling in random networks, Science 286 (5439) (1999) 509-512.

[42] L. Lovász, Random walks on graphs: A survey, Combinatorics, Paul Erdős is Eighty 2 (1) (1993) 1-46.

[43] R. Diestal, Graph Theory, Electronic Edition, Springer-Verlag, Heidelberg, 2005.

[44] M. Molloy, B. Reed, A critical point for random graphs with a given degree sequence, Random structures \& algorithms 6 (2-3) (1995) 161180.

[45] R. Rossi, N. Ahmed, The network data repository with interactive graph analytics and visualization, in: Twenty-Ninth AAAI Conference on Artificial Intelligence, 2015, pp. 4292-4293.

[46] T. M. J. Fruchterman, E. M. Reingold, Graph drawing by force-directed placement, Software: Practice and Experience 21 (11) (1991) 1129-1164.

[47] G. Bianconi, A.-L. Barabási, Competition and multiscaling in evolving networks, Europhysics Letters 54 (4) (2001) 436.

[48] M. S. Granovetter, The strength of weak ties, American Journal of Sociology 78 (6) (1977) 1360-1380.

[49] M. Bellingeri, D. Bevacqua, F. Scotognella, R. Alfieri, Q. Nguyen, D. Montepietra, D. Cassi, Link and node removal in real social networks: A review, Frontiers in Physics 8 (2020) 228.

[50] T. Antal, P. Krapivsky, Weight-driven growing networks, Physical Review E 71 (2) (2005) 026103. 